\title{
HITTING TIME OF LARGE SUBSETS OF THE HYPERCUBE
}

\author{
JIŘÍ ČERNÝ AND VÉRONIQUE GAYRARD
}

\begin{abstract}
We study the simple random walk on the $n$-dimensional hypercube, in particular its hitting times of large (possibly random) sets. We give simple conditions on these sets ensuring that the properly-rescaled hitting time is asymptotically exponentially distributed, uniformly in the starting position of the walk. These conditions are then verified for percolation clouds with densities that are much smaller than $(n \log n)^{-1}$. A main motivation behind this paper is the study of the so-called aging phenomenon in the Random Energy Model (REM), the simplest model of a meanfield spin glass. Our results allow us to prove aging in the REM for all temperatures, thereby extending earlier results to their optimal temperature domain.
\end{abstract}

\section{INTRODUCTION}

Let $\mathcal{V}_{n}$ be the $n$-dimensional hypercube, $\mathcal{V}_{n}=\{0,1\}^{n}$. We equip $\mathcal{V}_{n}$ with the metric

$$
d(x, y)=\sum_{i=1}^{n} \mathbb{1}\{x(i) \neq y(i)\},
$$

where $x(i)$ are the coordinates of $x \in \mathcal{V}_{n}$. Let further $Y_{n}$ be the simple random walk on $\mathcal{V}_{n}$. That is, $Y_{n}$ is the discrete-time Markov chain with state space $\mathcal{V}_{n}$ whose transition probabilities are given by $\mathbb{P}\left[Y_{n}(k+1)=y \mid Y_{n}(k)=x\right]=n^{-1}$ if $d(x, y)=1$, and 0 otherwise. We write $\mathbb{P}_{x}$ for the distribution of $Y_{n}$ conditioned on $Y_{n}(0)=x$. For $A \subset \mathcal{V}_{n}$ we define the hitting time of $A$ by

$$
H_{n}(A)=\min \left\{k \geq 0: Y_{n}(k) \in A\right\} .
$$

We are interested in the distribution of the hitting time of large random subsets of the hypercube. Specifically, let $\rho \in[0,1]$. We say that the set $A \subset \mathcal{V}_{n}$ is a percolation cloud on $\mathcal{V}_{n}$ with density $\rho$ if each site $x \in \mathcal{V}_{n}$ is in $A$ with probability $\rho$ independently of all others.

Our main aim is to prove the following theorem.

Theorem 1.1. Let $\bar{m}(n)$ be such that

$$
n \log n \ll \bar{m}(n) \ll 2^{n}(\log n)^{-1}
$$

and let $A_{n}$ be a sequence of percolation clouds on $\mathcal{V}_{n}$ with densities $\bar{m}(n)^{-1}$ defined on a common probability space $(\Omega, F, P)$. Then, for all $a>0$,

$$
\lim _{n \rightarrow \infty} \max _{x \in \mathcal{V}_{n}}\left|\mathbb{P}_{x}\left[H_{n}\left(A_{n} \backslash x\right) \geq a \bar{m}(n)\right]-e^{-a}\right|=0, \quad P \text {-a.s. }
$$

In words, the distribution of the normalised hitting time of $A_{n} \backslash x$ converges to the exponential distribution uniformly in the starting position $x$.

Date: November 8, 2018.

2000 Mathematics Subject Classification. 60J10, 60K37, 82D30.

Key words and phrases. Hitting time, random walk, hypercube, aging. 
In Theorem 1.2 below we state a similar result for another important class of random sets, namely sets $A_{n}$ that are sampled from $\mathcal{V}_{n}$ without replacement (i.e. each subset of $\left|A_{n}\right|$ elements of $\mathcal{V}_{n}$ is equally likely).

Theorem 1.2. Let $M_{n}$ be a sequence of integers such that, setting $m(n)=2^{n} / M_{n}$,

$$
n \log n \ll m(n) \leq 2^{n}
$$

Let $A_{n}$ be subsets of $M_{n}$ elements sampled from $\mathcal{V}_{n}$ without replacement, and defined on a common probability space $\left(\Omega^{\prime}, F^{\prime}, P^{\prime}\right)$. Then, for all $a>0$,

$$
\lim _{n \rightarrow \infty} \max _{x \in \mathcal{V}_{n}}\left|\mathbb{P}_{x}\left[H_{n}\left(A_{n} \backslash x\right) \geq \operatorname{am}(n)\right]-e^{-a}\right|=0, \quad P^{\prime} \text {-a.s. }
$$

Estimates on the distribution of the hitting time of various subsets of the hypercube have a long history. They can be traced back to the early literature on first passage times for Markov chains (see [Kem61] and the references therein) where these questions are reformulated in terms of the (one-dimensional) Ehrenfest urn scheme. These results provide very sharp estimates on the asymptotic distribution of the hitting time of a single point. More, recently Matthews [Mat89, p.118] gave finite- $n$ estimates for the Laplace transform of the hitting time of sets containing one or two points. These estimates are key ingredients that enter his description of the covering time of the hypercube and related questions.

The hitting times of much more general sets (possibly random, and whose size is possibly increasing with $n$ ) were studied very recently in [BČ06a] and [BG06]. In [BG06], Ben Arous and Gayrard give precise conditions for the hitting time of subsets of $\mathcal{V}_{n}$ to be asymptotically exponentially distributed for a class of subsets for which the so-called lumping construction can be applied. This construction was fist used in this context by BBG03a. It can be understood as a $d$-dimensional extension of the Ehrenfest urn scheme where the random walk $Y_{n}$ on the hypercube is replaced by a walk defined on a $d$-dimensional state space of smaller cardinality, and which evolves in a convex potential that is very steep near its boundary. Such a chain is then studied using the tools developed in [BEGK01, BEGK02] for the study of metastability in reversible Markov chains on discrete state space. This method allows in particular to show that Theorem 1.1 is valid for $m(n) \geq C 2^{n} / \log n$.

In [BČ06a], Ben Arous and Černý obtain a result similar to Theorem 1.1. Namely, they prove convergence to the exponential distribution of the hitting time of percolation clouds for densities of order $2^{-c n}$ with $c \in(3 / 4,1)$ (see Lemma 3.7 of [BČ06a]). The method is based on the formula discovered by Matthews in his study of covering problems (see Theorem 1.3 in [Mat88]) and on (improved) estimates from [Mat89.

The results of the present paper are the first that allow to treat sets of very large size, namely sets as large as $o\left(2^{n}(n \log n)^{-1}\right)$.

We will show in Section 4 that both Theorem 1.1 and Theorem 1.2 are consequences of the following more general theorem. To state it we define the function $\xi_{n}(k)$ by

$$
\xi_{n}(k)=2^{-n} \frac{n}{2}\left(\begin{array}{l}
n \\
k
\end{array}\right)^{-1} \sum_{j=1}^{n-k}\left(\begin{array}{c}
n \\
k+j
\end{array}\right) \frac{1}{j}, \quad k=0, \ldots, n .
$$

The role of this relatively complicated function will become evident in Lemma 3.6.

Theorem 1.3. Let $n \log n \ll m(n) \leq 2^{n}$ and $B_{n} \subset \mathcal{V}_{n}$ be such that

$$
0<\left|B_{n}\right|=2^{n} m(n)^{-1}(1+o(1)) \text {. }
$$


Define

$$
\begin{aligned}
v_{n}(k) & =\max _{x \in \mathcal{V}_{n}}\left|\left\{y \in B_{n}: d(x, y)=k\right\}\right|, \\
V_{n}(k) & =\max _{x \in \mathcal{V}_{n}}\left|\left\{y \in B_{n}: d(x, y) \leq k\right\}\right| .
\end{aligned}
$$

If there exists a function $g(n)$, such that $g(n) \leq n / 2$,

$$
\xi_{n}(g(n)) \ll 2^{-n} m(n),
$$

and

$$
\lim _{n \rightarrow \infty} \sum_{k=1}^{g(n)-1} v_{n}(k) \xi_{n}(k)=0, \quad \text { and } \quad V_{n}(g(n)-1) \ll\left|B_{n}\right|
$$

then

$$
\lim _{n \rightarrow \infty} \max _{x \in \mathcal{V}_{n}}\left|\mathbb{P}_{x}\left[H_{n}\left(B_{n} \backslash x\right) \geq a m(n)\right]-e^{-a}\right|=0 .
$$

Remark. 1. While the upper bound on $m(n)$ in the range of validity of the preceding theorem is trivially optimal, we cannot claim the same about the lower bound. Morally, the proof exploits the fact that the simple random walk on the hypercube equilibrates after $O(n \log n)$ steps, even if an application of this fact is not easy to see in our actual proof. It is intuitively obvious that after equilibration the hitting time should be exponentially distributed. The claim of the theorem might stay valid even for $m(n)=O(n \log n)$, however for different reasons. Several serious technical complications appears in our proof for such $m(n)$. We were not motivated to proceed further since it is not needed for the applications we have in mind.

2. The results of [BG06] (see Theorem 1.7 of [BG06] and the simpler Corollary 1.8) imply that Theorem 1.1 holds true for sets $B_{n}$ whose size is asymptotically constant that is, when (1.8) holds for $m(n)=2^{n} / M$ where $M$ is a fixed integer.

3. We will show soon that $\xi_{n}(1)=O\left(n^{-1}\right)$. Therefore, the conditions appearing in (1.11) are void when (1.8) holds with $m(n)$ satisfying $2^{-n} m(n) \gg n^{-1}$ (and, in particular, when $\left|B_{n}\right|$ is constant). As an immediate consequence of Theorem 1.3 we then obtain:

Corollary 1.4. Theorem 1.1 remains valid on $2^{n}(\log n)^{-1} \leq \bar{m}(n) \leq 2^{n}$ if the term $a \bar{m}(n)$ in (1.4) is replaced by $2^{n}\left|A_{n}\right|^{-1}$.

Proof. It is sufficient to take $m(n)$ in Theorem 1.3 to be $m(n)=2^{n} /\left|A_{n}\right|$, where $A_{n}$ is the percolation cloud with intensity $\bar{m}(n)$.

Our method of proof relies on a sharp estimate on the distribution of hitting time of a single point when time is measured on the scale $m(n)$, namely on the quantity

$$
\mathbb{P}_{z_{k}}\left[H_{n}(\mathbf{0})<a m(n)\right]
$$

where $\mathbf{0}$ is the vertex of $\mathcal{V}_{n}$ whose coordinates are all 0 and $z_{k}$ is any vertex at distance $k$ from it; this estimate is itself derived from an estimate on the Laplace transform of the hitting time $H_{n}(\mathbf{0})$ (see respectively Lemma 3.6 and Lemma 3.4). The domain of validity of the latter determines our bounds on $m(n)$. On this domain the Laplace transform is well approximated by the sum of two terms: the expected contribution of an exponential distribution, and the mysterious looking function $\xi_{n}(k)$ which is, essentially, the probability that $Y_{n}$ started from $z_{k}$ hits $\mathbf{0}$ in the first $n \log n$ steps.

The rest of the paper is organised as follows. In the next section we describe our main motivation and give some important consequences of our results for aging in 
the Random Energy model. In Section 3 we give the proof of Theorem 1.3. Finally, Theorems 1.1 and 1.2 are proved in Section 4 .

\section{Aging in the Random Energy Model}

The main motivation behind this paper originates in the study of the Random Hopping Time (RHT) dynamics of the Random Energy Model (REM), which is often called the simplest model of a spin glass. Let us describe this problem briefly (for a recent review see $[\mathrm{BC} 06 \mathrm{~b}])$. In the REM an energy $E_{x}$ is attached to every site $x \in \mathcal{V}_{n}$. The $E_{x}$ 's are chosen to be i.i.d. with standard normal distribution. Given a collection $\boldsymbol{E}=\left\{E_{x}: x \in \mathcal{V}_{n}\right\}$ and a parameter $\beta>0$ (representing the inverse of the temperature), the RHT dynamics in the REM is defined as a continuous-time Markov process $X_{n}(\cdot)$ whose transition rates are given by $w_{n}^{\boldsymbol{E}}(x, y)=\exp \left(-\beta \sqrt{n} E_{x}\right)$ if $d(x, y)=1$ and zero otherwise.

The main goal of the study of the processes $X_{n}$ was to prove aging. In this context it usually means showing that the two-point function

$$
R_{n}\left(t_{w}, t_{w}+t ; \boldsymbol{E}\right):=P\left[X_{n}\left(t_{w}+t\right)=X_{n}\left(t_{w}\right) \mid \boldsymbol{E}\right],
$$

has a non-trivial limit as $t_{w}, t=\theta t_{w}$ and $n$ tend simultaneously to infinity.

The first proof of aging in the REM for $\beta>\beta_{c}=\sqrt{2 \log 2}$ and times $t_{w} \sim \exp \left(\beta \beta_{c} n\right)$ was given in BBG03a, BBG03b, based on the analysis of the metastable behaviour of $X_{n}$ and renewal theory. In [BČ06a another proof of aging, based on the arc-sine law for stable subordinators, was given for temperatures satisfying

$$
\sqrt{3 / 4}<\alpha \beta / \beta_{c}<1 \text {. }
$$

and (shorter) time-scales $t_{w}(n)=(\alpha \beta \sqrt{2 \pi n})^{-1 / \alpha} \exp \left(\alpha \beta^{2} n\right)$, where $\alpha \in(0,1)$ is a free parameter. We will now explain how our methods allows to improve the lower bound $\sqrt{3 / 4}$ in (2.2) to its optimal value, that is to 0 . To this end we need to describe briefly the background of the techniques of [BČ06a].

The transition rates of the process $X$ do not depend on the energy of the target vertex $y$. Therefore, the process $X_{n}$ is a time change of the simple random walk $Y_{n}$, and can be written as $X_{n}(t)=Y_{n}\left(S_{n}^{-1}(t)\right)$, where

$$
S_{n}(k)=\sum_{i=0}^{k-1} e_{i} \exp \left(\beta \sqrt{n} E_{Y_{n}(i)}\right),
$$

$S_{n}^{-1}$ is the generalised right-continuous inverse of $S_{n}(k)$, and $e_{i}$ is a sequence of meanone i.i.d. exponential random variables. $S_{n}(k)$ is the time of the $k^{\text {th }}$ jump of $X_{n}$.

It was proved in BČ $\mathrm{B}$ a that $S(n)$ behaves (for $n$ large) as an $\alpha$-stable subordinator in certain time and temperature regimes. To be more precise, set $r(n)=\exp \left(\alpha^{2} \beta^{2} n / 2\right)$. Then, if (2.2) holds then $t(n)^{-1} S_{n}(\lfloor r(n) \cdot\rfloor)$ converges in distribution to an $\alpha$-stable subordinator.

It is a known fact that the value of a stable subordinator at time $t$ can be approximated by the finite sum of its largest jumps up to this time. The same is true for $S_{n}$ : the main contribution to $S_{n}(r(n))$ comes from a finite number of visits to sites with $\exp \left(\beta \sqrt{n} E_{x}\right) \asymp t(n)$. Such sites form (due to the i.i.d. property of the energies) a percolation cloud. To understand properties of $S_{n}$ it is therefore necessary to understand how the simple random walk visits such clouds. 
As we have already remarked, the methods of [BČ06a] are sufficient to show Theorem 1.1 for densities much smaller than $2^{-3 n / 4}$. The constant $3 / 4$ in the exponent entails the constant 3/4 in (2.2). The result of our Theorem 1.1 thus allows to extend the domain of validity of Theorem 3.1 in $\mathrm{BC} 06 \mathrm{a}$. As we find this extension important we state it here:

Theorem 2.1. For $\alpha \in(0,1)$ let $t_{w}(n)$ and $r(n)$ be as above. If

$$
0<\alpha \beta<\beta_{c},
$$

then $t_{w}(n)^{-1} S_{n}(r(n) \cdot)$ converges in distribution to an $\alpha$-stable subordinator and the two-point function $R_{n}$ exhibits aging. Namely, for a.e. realisation of $\boldsymbol{E}$ and for every $\theta \in(0, \infty)$

$$
\lim _{n \rightarrow \infty} R_{n}\left(t_{w}(n),(1+\theta) t_{w}(n) ; \boldsymbol{E}\right)=\operatorname{Asl}_{\alpha}(1 / 1+\theta),
$$

where $\operatorname{Asl}_{\alpha}(u)$ stands for the distribution function of the generalised arcsine law with parameter $\alpha, \operatorname{Asl}_{\alpha}(z):=\pi^{-1} \sin \alpha \pi \int_{0}^{z} u^{\alpha-1}(1-u)^{-\alpha} \mathrm{d} u$.

It is worth noting that for any $\beta<\infty$ there exists $\alpha \in(0,1)$ such that (2.4) is satisfied. This implies that aging occurs in the RHT dynamics of the REM at all temperatures.

Theorem 1.1 allows further to study the RHT dynamics in another interesting timetemperature regime where denser percolation clouds should be considered: for $t_{w}(n)=$ $(\beta \sqrt{2 \pi n})^{-1} \exp \left(\beta^{2} n\right)$, that is for $\alpha=1$. It is argued in the physics literature [BB02] that the two-point function $R_{n}$ exhibits some interesting ultrametric behaviour in this case. The rigorous treatment of this problem was the original motivation behind this paper and will be subject of a forthcoming paper.

\section{Proof of Theorem 1.3}

As mentioned earlier Theorem 1.3 is already known for small sets, namely when $m(n)=2^{n} / M$ for $M$ a fixed integer (see [BG06], Corollary 1.8). Although our method of proof clearly allows us to cover this case as well, its treatment is, in places, quite different from the case $n \log n \ll m(n) \ll 2^{n}$. Thus, in order to keep this paper as concise as possible we will prove Theorem 1.3 in that latter case only and assume from now on that $n \log n \ll m(n) \ll 2^{n}$.

Proof of Theorem 1.3 ( for $\left.n \log n \ll m(n) \ll 2^{n}\right)$. We have

$$
\mathbb{P}_{x}\left[H_{n}\left(B_{n} \backslash x\right) \geq a m(n)\right]=1-\mathbb{P}_{x}\left[\bigcup_{y \in B_{n} \backslash x} H_{n}(y)<a m(n)\right] .
$$

Therefore, it follows from the inclusion-exclusion principle that for all even $\ell \in \mathbb{N}$

$$
\mathbb{P}_{x}\left[H_{n}\left(B_{n} \backslash x\right) \geq a m(n)\right] \leq 1+\sum_{i=1}^{\ell} \frac{(-1)^{i}}{i !} \sum_{y_{1}, \ldots, y_{i} \in B_{n} \backslash x}^{\prime} \mathbb{P}_{x}\left[\bigcap_{j=1}^{i} H_{n}\left(y_{j}\right)<a m(n)\right],
$$

where $\sum^{\prime}$ denotes the sum over all mutually different $y$ 's. Analogous expressions for $\ell$ odd give lower bounds.

The following proposition is the key step of the proof. 
Proposition 3.1. Under the assumptions of Theorem 1.3 and assuming that $n \log n \ll$ $m(n) \ll 2^{n}$ we have, for all $i \in \mathbb{N}$,

$$
\lim _{n \rightarrow \infty} \max _{x \in \mathcal{V}_{n}}\left|\sum_{y_{1}, \ldots, y_{i} \in B_{n} \backslash x}^{\prime} \mathbb{P}_{x}\left[\bigcap_{j=1}^{i} H_{n}\left(y_{j}\right)<a m(n)\right]-a^{i}\right|=0 .
$$

Using Proposition 3.1 the completion of the proof of Theorem 1.3 under the assumption that $n \log n \ll m(n) \ll 2^{n}$ is immediate.

The proof of Proposition 3.1 relies on several technical lemmas which we collect in the subsection below. The proof of Proposition 3.1 is then concluded in subsection 3.2 .

3.1. Preparatory Lemmas. Our first lemma collects the properties of the function $\xi_{n}(k)$ which will be needed later.

Lemma 3.2. (i) For all $k \in\{1, \ldots, n\}, \xi_{n}(k) \leq K\left(\begin{array}{l}n \\ k\end{array}\right)^{-1} n^{1 / 2} \log n$, where $K$ is a constant independent of $n$ and $k$.

(ii) For all $k \leq n / 2, \xi_{n}(k) \geq \frac{1}{2}\left(\begin{array}{l}n \\ k\end{array}\right)^{-1}$.

(iii) For any fixed $n$ the function $\xi_{n}(k)$ is decreasing in $k$.

(iv) If $k=o(n)$, then $\xi_{n}(k)=\left(\begin{array}{l}n \\ k\end{array}\right)^{-1}(1+o(1))$.

Proof. (i) Recall that $\xi_{n}(k)=2^{-n} \frac{n}{2}\left(\begin{array}{l}n \\ k\end{array}\right)^{-1} \sum_{j=1}^{n-k}\left(\begin{array}{c}n \\ j+k\end{array}\right) \frac{1}{j}$. From a standard moderate deviations argument it follows that

$$
2^{-n} \frac{n}{2} \sum_{\substack{j=0 \\
|j-n / 2| \geq n^{7 / 12}}}^{n}\left(\begin{array}{l}
n \\
j
\end{array}\right) \leq c_{1} \frac{n}{2} e^{-c_{2} n^{1 / 6}}
$$

Therefore, the contribution of $j$ 's with $|k+j-n / 2| \geq n^{7 / 12}$ is $o\left(\left(\begin{array}{l}n \\ k\end{array}\right)^{-1}\right)$. For the remaining $j$ 's we use the approximation

$$
\left(\begin{array}{c}
n \\
n / 2+i
\end{array}\right)=\sqrt{\frac{2}{\pi}} n^{-1 / 2} 2^{n} e^{-2 i^{2} / n}(1+o(1)),
$$

which is valid uniformly for $i=o\left(n^{2 / 3}\right)$. Setting $a=n^{-1 / 2}(k+j-n / 2)$ and $b=n^{-1 / 2}(k-$ $n / 2)$, and thus $j=n^{1 / 2}(a-b)$, the contribution of the $j$ 's with $|k+j-n / 2| \leq n^{7 / 12}$ to $\xi_{n}(k)$ equals

$$
\begin{aligned}
& 2^{-n} \frac{n}{2}\left(\begin{array}{l}
n \\
k
\end{array}\right)^{-1} \sum_{\substack{a \in\left[-n^{1 / 12}, n^{1 / 12}\right] \cap(\mathbb{Z} / \sqrt{n}) \\
a \geq b+n^{-1 / 2}}}\left(\begin{array}{c}
n \\
a \sqrt{n}+n / 2
\end{array}\right) \frac{1}{(a-b) \sqrt{n}} \\
& \leq K^{\prime} n^{1 / 2}\left(\begin{array}{l}
n \\
k
\end{array}\right)^{-1} \int_{\left(b+n^{-1 / 2}\right) \vee-n^{1 / 12}}^{n^{1 / 12}} e^{-2 x^{2}} \frac{\mathrm{d} x}{(x-b)} \leq K\left(\begin{array}{l}
n \\
k
\end{array}\right)^{-1} n^{1 / 2} \log n .
\end{aligned}
$$

(ii) For $k<n / 2, \xi_{n}(k) \geq 2^{-n} \frac{n}{2}\left(\begin{array}{l}n \\ k\end{array}\right)^{-1} \sum_{j=1}^{n / 2}\left(\begin{array}{c}n \\ k+j\end{array}\right) \frac{2}{n} \geq \frac{1}{2}\left(\begin{array}{l}n \\ k\end{array}\right)^{-1}$.

(iii) The function $\xi_{n}$ can be rewritten as

$$
\xi_{n}(k)=2^{-n} \frac{n}{2} \sum_{j=1}^{n-k}\left(\begin{array}{c}
n-k \\
j
\end{array}\right)\left(\begin{array}{c}
k+j \\
j
\end{array}\right)^{-1} \frac{1}{j} .
$$

Here, the fact that $\xi_{n}(k)$ is decreasing is apparent. 
(iv) Using again the moderate deviations argument, for $k=o(n)$,

$$
\xi_{n}(k)=2^{-n} \frac{n}{2}\left(\begin{array}{l}
n \\
k
\end{array}\right)^{-1} \sum_{j=n / 2-n^{7 / 12}-k}^{n / 2+n^{7 / 12}-k}\left(\begin{array}{c}
n \\
j+k
\end{array}\right) \frac{1}{j}(1+o(1))=\left(\begin{array}{l}
n \\
k
\end{array}\right)^{-1}(1+o(1)) .
$$

This completes the proof of Lemma 3.2 .

We now prove that for $m(n)$ in the range considered in Theorem 1.3 there always exists a function $g(n)$ with $g(n) \leq n / 2$ satisfying (1.10). We in fact prove a little more:

Lemma 3.3. Let $m(n)$ be such that $n \log n \ll m(n) \ll 2^{n}$. Then there exist function $g$ such that

$$
n / 2-g(n) \gg n^{1 / 2} \quad \text { and } \quad \xi_{n}(g(n)) \ll 2^{-n} m(n) .
$$

Proof. Take $m^{\prime}(n)$ such that $n \log n \ll m^{\prime}(n) \ll m(n)$ and define $g(n)$ by

$$
g(n)=\min \left\{k: \xi_{n}(k) \leq 2^{-n} m^{\prime}(n)\right\} .
$$

Such $g(n)$ satisfies the second half of (3.9) by definition. By Lemma 3.2)(i)

$$
g(n) \leq \min \left\{k: K\left(\begin{array}{l}
n \\
k
\end{array}\right)^{-1} n^{1 / 2} \log n \leq 2^{-n} m^{\prime}(n)\right\}
$$

Write $k=n / 2-i$. Using formula (3.5) for the binomial coefficient we find that

$$
g(n) \leq \frac{n}{2}+\min \left\{i: e^{2 i^{2} / n} \leq \frac{c m^{\prime}(n)}{n \log n}\right\} .
$$

Since $m^{\prime}(n) \gg n \log n$ there exists $i_{0}, n^{2 / 3} \gg i_{0} \gg n^{1 / 2}$, for which the inequality in braces holds. Since $n / 2-g(n) \geq i_{0}$, the first half of $(3.9)$ is proved.

Let us assume from now on that $g(n)$ satisfies (3.9). As announced earlier, the key ingredient of the proof of Proposition 3.1 is a precise estimate on the Laplace transform of the hitting time of a single point. We now state and prove this result. As we will see, this is where the function $\xi_{n}$ comes in.

Lemma 3.4. Let $\mathbf{0}$ be the vertex of the hypercube with all coordinates equal to 0 and let $z_{k} \in \mathcal{V}_{n}$ be an arbitrary vertex of the hypercube such that $d\left(z_{k}, \mathbf{0}\right)=k$. If $n \log n \ll$ $m(n) \ll 2^{n}$, then for all $s>0$

$$
\mathbb{E}_{z_{k}} \exp \left(-\frac{s}{m(n)} H_{n}(\mathbf{0})\right)=\left[2^{-n} \frac{m(n)}{s}+\xi_{n}(k)\right](1+o(1)) .
$$

Proof. By Fourier methods for random walks on finite groups [Dia88], we have as in Mat89, BČ06a

$$
\mathbb{E}_{z_{k}} e^{-s / m H_{n}(\mathbf{0})}=\frac{\sum_{y \in \mathcal{V}_{n}}(-1)^{z_{k} \cdot y}\left[1-e^{-s / m}\left(1-\frac{2 d(y, \mathbf{0})}{n}\right)\right]^{-1}}{\sum_{y \in \mathcal{V}_{n}}\left[1-e^{-s / m}\left(1-\frac{2 d(y, \mathbf{0})}{n}\right)\right]^{-1}},
$$

where $x \cdot y=\sum_{i=1}^{n} x(i) y(i)$ is the standard scalar product in $\mathbb{R}^{n}$.

Let us first consider the numerator of (3.14) . Observe that there are $\left(\begin{array}{c}k \\ i\end{array}\right)\left(\begin{array}{c}n-k \\ j\end{array}\right)$ sites $y \in \mathcal{V}_{n}$ such that $d(0, y)=i+j$ and $z_{k} \cdot y=i$. Hence the numerator of (3.14) is equal to

$$
\sum_{i=0}^{k} \sum_{j=0}^{n-k}(-1)^{i}\left(\begin{array}{l}
k \\
i
\end{array}\right)\left(\begin{array}{c}
n-k \\
j
\end{array}\right) \frac{1}{1-e^{-s / m}\left(1-2 n^{-1}(i+j)\right)} .
$$

This expression can be simplified using the following lemma. 
Lemma 3.5. For all $k, j \in\{0,1, \ldots\}$ and all $s>0$

$$
\sum_{i=0}^{k} \frac{(-1)^{i}\left(\begin{array}{l}
k \\
i
\end{array}\right)}{1-e^{-s / m}\left(1-2 n^{-1}(i+j)\right)}=\frac{n e^{s / m}}{2} \cdot \frac{\Gamma(1+k) \Gamma\left(j+\frac{n}{2}\left(e^{s / m}-1\right)\right)}{\Gamma\left(1+k+j+\frac{n}{2}\left(e^{s / m}-1\right)\right)} .
$$

Proof. Note that the second fraction on the right-hand side of (3.16) can be expressed using the Beta-integral,

$$
\frac{n e^{s / m}}{2} \cdot \frac{\Gamma(1+k) \Gamma\left(j+\frac{n}{2}\left(e^{s / m}-1\right)\right)}{\Gamma\left(1+k+j+\frac{n}{2}\left(e^{s / m}-1\right)\right)}=\frac{n e^{s / m}}{2} \int_{0}^{1}(1-t)^{k} t^{i+\frac{n}{2}\left(e^{s / m}-1\right)-1} \mathrm{~d} t .
$$

Expanding $(1-t)^{k}$ according to the binomial theorem, and performing an easy integration then gives the left-hand side of (3.16).

Using the last lemma the numerator of (3.14) can be rewritten as

$$
\sum_{j=0}^{n-k}\left(\begin{array}{c}
n-k \\
j
\end{array}\right) \frac{n e^{s / m}}{2} \cdot \frac{\Gamma(1+k) \Gamma\left(j+\frac{n}{2}\left(e^{s / m}-1\right)\right)}{\Gamma\left(1+k+j+\frac{n}{2}\left(e^{s / m}-1\right)\right)} .
$$

So far we obtained an exact expression which we now want to evaluate. To do so we will use the following known properties of $\Gamma$-functions (we refer to [AS72] for the definition and the properties of the functions appearing below).

$$
\Gamma^{\prime}(x)=\Gamma(x) \psi_{0}(x), \quad \Gamma^{\prime \prime}(x)=\Gamma(x)\left(\psi_{0}(x)^{2}+\psi_{1}(x)\right), \quad \lim _{x \rightarrow 0} x \Gamma(x)=1,
$$

where $\psi_{0}$ is the digamma function and $\psi_{1}=\psi_{0}^{\prime}$. The values of these functions for integer arguments can be written explicitly:

$$
\psi_{0}(k)=\sum_{i=1}^{k-1} \frac{1}{i}-\gamma_{E}=\log k(1+o(1)), \quad \psi_{1}(k)=\frac{\pi^{2}}{6}-\sum_{i=1}^{k-1} \frac{1}{i^{2}}=O(1 / k) .
$$

where $\gamma_{E}$ is Euler's constant.

We can now evaluate (3.18). Set $\varepsilon=\frac{n}{2}\left(e^{s / m}-1\right)$ and observe that the bound $m \gg n \log n$ entails $\varepsilon \ll(\log n)^{-1}$. To treat the term $j=0$ in (3.18) simply note that, since $\varepsilon \psi_{0}(1+k)=o(1)$ for all $k \leq n$,

$$
\frac{n e^{s / m}}{2} \cdot \frac{\Gamma(1+k) \Gamma(\varepsilon)}{\Gamma(1+k+\varepsilon)}=\frac{n e^{s / m}}{2} \cdot \frac{\varepsilon^{-1}(1+o(1))}{1+\varepsilon \psi_{0}(1+k)}=\frac{m}{s}(1+o(1)) .
$$

A similar calculation for the remaining terms combined with (3.7) readily yields

$$
\begin{aligned}
& \sum_{j=1}^{n-k}\left(\begin{array}{c}
n-k \\
j
\end{array}\right) \frac{n e^{s / m}}{2} \cdot \frac{\Gamma(1+k) \Gamma(j+\varepsilon)}{\Gamma(1+k+j+\varepsilon)} \\
& \quad=\sum_{j=1}^{n-k} \frac{n}{2 j}\left(\begin{array}{c}
n-k \\
j
\end{array}\right)\left(\begin{array}{c}
k+j \\
j
\end{array}\right)^{-1} \frac{\left(1+\varepsilon \psi_{0}(j)\right)(1+o(1))}{1+\varepsilon \psi_{0}(1+k+j)}=2^{n} \xi_{n}(k)(1+o(1)) .
\end{aligned}
$$

For $k=0$ the numerator of (3.14) coincides with the denominator. Equations (3.21), (3.22) and Lemma 3.2(iv) then imply that the denominator behaves like

$$
\left\{m s^{-1}+2^{n} \xi_{n}(0)\right\}(1+o(1))=2^{n}(1+o(1)) .
$$

Finally, putting together (3.21), (3.22) and (3.23) yields the claim of Lemma 3.4 . 
Lemma 3.4 now allows us to get information on the form of the probability distribution function of $H_{n}(\mathbf{0})$. Let us denote by $p_{n}(a, k)$ the probability

$$
p_{n}(a, k)=\mathbb{P}_{z_{k}}\left[H_{n}(\mathbf{0})<a m(n)\right] .
$$

Lemma 3.6. (i) There exists $C<\infty$ independent of $n$ and $k$ such that

$$
p_{n}(a, k) \leq C e^{a}\left(2^{-n} m(n)+\xi_{n}(k)\right) .
$$

(ii) For any $a \in[0, \infty)$, uniformly on compact subsets of this interval,

$$
\lim _{n \rightarrow \infty} \max _{k \geq g(n)}\left|2^{n} m(n)^{-1} p_{n}(a, k)-a\right|=0 .
$$

Proof. Assertion (i) follows from Chebyshev inequality and Lemma 3.4. To prove (ii) observe that for $k \geq g(n)$, by (3.9) and by Lemma 3.2(iii), $\xi_{n}(k) \leq \xi_{n}(g(n)) \ll$ $2^{-n} m(n)$. Therefore, $\mathbb{E}_{z_{k}} \exp \left(-s H_{n}(\mathbf{0}) / m(n)\right)=2^{-n} m(n) / s(1+o(1))$. Consider the sequence of measures $\mu_{n}$ given by

$$
\mu_{n}([0, t])=2^{n} m(n)^{-1} \mathbb{P}_{z_{k}}\left[H_{n}(\mathbf{0}) / m(n) \in[0, t]\right] .
$$

The Laplace transform of $\mu_{n}$ then satisfies $\int_{0}^{\infty} e^{-s t} \mu_{n}(\mathrm{~d} t) \stackrel{n \rightarrow \infty}{\longrightarrow} 1 / s$. Therefore, $\mu_{n}$ converges weakly to the Lebesgue measure (see [Fel71], Section XIII.1, Theorem 2a, p. 433) and thus

$$
2^{n} m(n)^{-1} p_{n}(a, k)=\mu_{n}([0, a)) \stackrel{n \rightarrow \infty}{\longrightarrow} a .
$$

The uniformity on compact sets follows easily from the fact that the probabilities $p_{n}(a, k)$ are increasing in $a$.

We finally use Lemma 3.6 to get information on the form of the probability distribution function of the hitting time of finite subsets of points of $\mathcal{V}_{n}$. For $y_{1}, \ldots, y_{\ell} \in \mathcal{V}_{n}$ we define $\bar{H}_{n}\left(y_{1}, \ldots, y_{\ell}\right)$ by

$$
\bar{H}_{n}\left(y_{1}, \ldots, y_{\ell}\right)= \begin{cases}H_{n}\left(y_{\ell}\right) & \text { if } H_{n}\left(y_{i}\right)<H_{n}\left(y_{i+1}\right) \text { for all } i \in\{1, \ldots, \ell-1\} \\ \infty & \text { otherwise }\end{cases}
$$

That is $\bar{H}_{n}\left(y_{1}, \ldots, y_{\ell}\right)$ is finite only if the $y$ 's were visited in the prescribed order. In this case it is equal to the time to visit all $y$ 's. Observe that it is always infinite if $y_{i}=y_{j}$ for some $i \neq j$.

Lemma 3.7. Let $x=y_{0}$, let $y_{1}, \ldots, y_{\ell}$ be mutually distinct points in $\mathcal{V}_{n}$, and let $d(i)=d\left(y_{i-1}, y_{i}\right)$. Then

$$
\mathbb{P}_{x}\left[\bar{H}_{n}\left(y_{1}, \ldots, y_{\ell}\right)<a m(n)\right] \leq C^{\ell} e^{\ell a} \prod_{i=1}^{\ell}\left(2^{-n} m(n)+\xi_{n}(d(i))\right) .
$$

Proof. Obviously, by the strong Markov property,

$$
\mathbb{P}_{x}\left[\bar{H}_{n}\left(y_{1}, \ldots, y_{\ell}\right)<a m(n)\right] \leq \prod_{i=1}^{\ell} \mathbb{P}_{y_{i-1}}\left[H_{n}\left(y_{i}\right)<a m(n)\right],
$$

which, by Lemma 3.6(i), is bounded by the right-hand side of (3.30).

Lemma 3.8. Let $x^{n}=y_{0}^{n}$, let $y_{1}^{n}, \ldots, y_{\ell}^{n} \in \mathcal{V}_{n}$, and let $d_{n}(i)=d\left(y_{i}^{n}, y_{i-1}^{n}\right)$. Suppose that $d_{n}(i) \geq g(n)$ for all $i \in\{1, \ldots, \ell\}$ and all $n$. Then, uniformly over all $x^{n}$ and $y_{i}^{n}$,

$$
\lim _{n \rightarrow \infty} 2^{\ell n} m(n)^{-\ell} \mathbb{P}_{x^{n}}\left[\bar{H}_{n}\left(y_{1}^{n}, \ldots, y_{\ell}^{n}\right)<a m(n)\right]=\frac{a^{\ell}}{\ell !} .
$$


Proof. The probability in question can be bounded from above using the strong Markov property,

$$
\mathbb{P}_{x^{n}}\left[\bar{H}_{n}\left(y_{1}^{n}, \ldots, y_{\ell}^{n}\right)<a m(n)\right] \leq \sum_{\substack{a_{1}, \ldots, a_{\ell} \in \mathbb{N} / m(n) \\ a_{1}+\cdots+a_{\ell}<a}} \prod_{i=1}^{\ell} \mathbb{P}_{y_{i-1}}\left[H_{n}\left(y_{i}\right) / m(n)=a_{i}\right]
$$

Since $d\left(y_{i-1}, y_{i}\right)>g(n)$, it is easy to see from Lemma 3.6(ii) that the sum behaves like

$$
2^{-n \ell} m(n)^{\ell} \int_{x_{1}+\cdots+x_{\ell}<a} \ldots \int_{1} \mathrm{~d} x_{1} \ldots \mathrm{d} x_{\ell}(1+o(1))=2^{-n \ell} m(n)^{\ell} a^{\ell} / \ell !(1+o(1)) .
$$

The above expression only provides an upper bound since it does not exclude the possibility that the random walk visits another $y_{j}, j>i$, on its way from $y_{i-1}$ to $y_{i}$. To construct a lower bound we should exclude such visits. Therefore, denoting by UB the upper bound (3.33),

$$
\begin{aligned}
& \mathbb{P}_{x^{n}}\left[\bar{H}_{n}\left(y_{1}^{n}, \ldots, y_{\ell}^{n}\right)<a m(n)\right] \\
& \geq \mathrm{UB}-\sum_{i=1}^{\ell} \sum_{j=i+1}^{\ell} \sum_{\substack{a_{1}, \ldots, a_{\ell} \in \mathbb{N} / m(n) \\
a_{1}+\cdots+a_{\ell}<a}} \prod_{\substack{k=1 \\
k \neq i}}^{\ell} \mathbb{P}_{y_{k-1}}\left[H_{n}\left(y_{k}\right) / m(n)=a_{k}\right] \\
& \quad \times \sum_{\substack{b \in \mathbb{N} / m(n) \\
b \in\left(0, a_{i}-a_{i-1}\right)}} \mathbb{P}_{y_{i-1}}\left[H_{n}\left(y_{j}\right) / m(n)=b\right] \mathbb{P}_{y_{j}}\left[H_{n}\left(y_{i}\right) / m(n)=a_{i}-b\right] .
\end{aligned}
$$

The negative term on the right-hand side is smaller than

$$
C 2^{-n(\ell-1)} m(n)^{\ell-1} e^{(\ell-1) a} \sum_{i=1}^{\ell} \sum_{j=i+1}^{\ell} \mathbb{P}_{y_{i-1}}\left[H_{n}\left(y_{j}\right)<a m(n)\right] \mathbb{P}_{y_{j}}\left[H_{n}\left(y_{i}\right)<a m(n)\right] .
$$

Hence, if we can show that for all $j \neq i$

$$
\mathbb{P}_{y_{i-1}}\left[H_{n}\left(y_{j}\right)<a m(n)\right] \mathbb{P}_{y_{j}}\left[H_{n}\left(y_{i}\right)<a m(n)\right]=o\left(2^{-n} m(n)\right),
$$

then the proof of Lemma 3.8 is finished.

Let $k=d\left(y_{i-1}, y_{j}\right)$ and $l=d\left(y_{j}, y_{i}\right)$. Since $d\left(y_{i-1}, y_{i}\right) \geq g(n)$ we have also $k+l \geq g(n)$. Obviously $k \geq 1, l \geq 1$. By Lemma 3.6(i),

$$
\begin{aligned}
\mathbb{P}_{y_{i-1}} & {\left[H_{n}\left(y_{j}\right)<a m(n)\right] \mathbb{P}_{y_{j}}\left[H_{n}\left(y_{i}\right)<a m(n)\right] } \\
& \leq C^{2} e^{2 a}\left(2^{-n} m(n)+\xi_{n}(k)\right) \cdot\left(2^{-n} m(n)+\xi_{n}(l)\right) \\
& \leq C^{2} e^{2 a}\left(2^{-2 n} m(n)^{2}+2^{-n} m(n) 2 \xi_{n}(1)+\xi_{n}(k) \xi_{n}(l)\right) .
\end{aligned}
$$

The first two summands are $o\left(2^{-n} m(n)\right)$ (see Lemma 3.2(iii,iv)). If $k$ or $l$ is larger than $g(n)$, then the same is valid for the third one. As the last step of the proof we show that if $\max \{k, l\}<g(n)$ and $k+l \geq g(n)$, then for any $\varepsilon>0$ and $n$ large enough

$$
\xi_{n}(k) \xi_{n}(l) \leq \varepsilon 2^{-n} m(n) .
$$

Let $z_{k+l}$ be as in Lemma 3.4 and let $z_{k}$ be any point such that $d\left(z_{k}, \mathbf{0}\right)=k$ and $d\left(z_{k}, z_{k+l}\right)=l$. Since on the way from $z_{k+l}$ to $\mathbf{0}$, the random walk may pass through $z_{k}$ we have

$$
\mathbb{E}_{z_{k+l}}\left[e^{-s H_{n}(\mathbf{0})}\right] \geq \mathbb{E}_{z_{k+l}}\left[e^{-s H_{n}\left(z_{k}\right)}\right] \mathbb{E}_{z_{k}}\left[e^{-s H_{n}(\mathbf{0})}\right]
$$


Lemma 3.4 then yields

$$
\left\{2^{-n} \frac{m(n)}{s}+\xi_{n}(k+l)\right\}(1+o(1)) \geq\left\{2^{-n} \frac{m(n)}{s}+\xi_{n}(k)\right\}\left\{2^{-n} \frac{m(n)}{s}+\xi_{n}(l)\right\} \geq \xi_{n}(k) \xi_{n}(l) .
$$

Since $k+l \geq g(n)$ we can, in view of (3.9), ignore the term $\xi_{n}(k+l)$ on the left-hand side. Taking $s$ sufficiently large then proves (3.39). This concludes the proof of the lemma.

We are now ready to complete the proof of Proposition 3.1 .

\subsection{Proof of Proposition 3.1. We shall establish that}

$$
\lim _{n \rightarrow \infty} \max _{x \in \mathcal{V}_{n}}\left|\sum_{y_{1}, \ldots, y_{i} \in B_{n} \backslash x}^{\prime} \mathbb{P}_{x}\left[\bigcap_{j=1}^{i} H_{n}\left(y_{j}\right)<a m(n)\right]-a^{i}\right|=0 .
$$

Observe that, with $y_{0}=x$,

$$
\begin{gathered}
\sum_{y_{1}, \ldots, y_{i} \in B_{n} \backslash x}^{\prime} \mathbb{P}_{x}\left[\bigcap_{j=1}^{i} H_{n}\left(y_{j}\right)<a m(n)\right]=i ! \sum_{y_{1}, \ldots, y_{i} \in B_{n} \backslash x}^{\prime} \mathbb{P}_{x}\left[\bar{H}_{n}\left(y_{1}, \ldots, y_{i}\right)<a m(n)\right] \\
=i ! \sum_{d_{1}, \ldots, d_{i}=1}^{n} \sum_{\substack{y_{1}, \ldots, y_{i} \in B_{n} \\
d\left(y_{i}, y_{i-1}\right)=d_{i}}}^{\prime} \mathbb{P}_{x}\left[\bar{H}_{n}\left(y_{1}, \ldots, y_{i}\right)<a m(n)\right] .
\end{gathered}
$$

Consider first the summation over distances larger than $g(n)$. Using Lemma 3.8 we get that (uniformly in the starting position $x$ )

$$
\begin{aligned}
i ! \sum_{d_{1}, \ldots, d_{i}=g(n)}^{n} & \sum_{\substack{y_{1}, \ldots, y_{i} \in B_{n} \\
d\left(y_{i}, y_{i-1}\right)=d_{i}}}^{\prime} \mathbb{P}_{x}\left[\bar{H}_{n}\left(y_{1}, \ldots, y_{i}\right)<a m(n)\right] \\
= & \sum_{\substack{d_{1}, \ldots, d_{i}=g(n) \\
y_{1}, \ldots, y_{i} \in B_{n} \\
d\left(y_{i}, y_{i-1}\right)=d_{i}}}^{\prime} 2^{-i n} m(n)^{i} a^{i}(1+o(1))=a^{i}(1+o(1)) .
\end{aligned}
$$

For the second equality we used the fact that by (1.8), (1.11) and the finiteness of $i$ there are $2^{n} m(n)^{-1}(1+o(1))$ choices for every $y_{i}$.

To estimate the remaining contribution to (3.43), we first bound the sum

$$
\sum_{d=1}^{g(n)-1} \sum_{y \in B_{n}: d(y, x)=d} C e^{a}\left(2^{-n} m(n)+\xi_{n}(d)\right) \leq \sum_{d=1}^{g(n)-1} v_{n}(d) \xi_{n}(d)+V_{n}(g(n)-1) 2^{-n} m(n) .
$$

Both summands of in the last formula converge to 0 which can be seen easily from (1.11) and (1.8). For $d>g(n)$, by Lemma 3.2(iii), $\xi_{d}(n) \ll 2^{-n} m(n)$. Therefore, using (1.8), for all $n$ large enough

$$
\sum_{d=1}^{n} \sum_{y \in B_{n}: d(y, x)=d} C e^{a}\left(2^{-n} m(n)+\xi_{n}(d)\right) \leq 2 C e^{a}\left|B_{n}\right| 2^{-n} m(n) \leq 4 C e^{a} .
$$


According to Lemma 3.7, the remaining part of (3.43) then satisfies

$$
\begin{aligned}
& i ! \sum_{\substack{d_{1}, \ldots, d_{i}=1 \\
\exists d_{i} \leq g(n)}}^{n} \sum_{\substack{y_{1}, \ldots, y_{i} \in B_{n} \\
d\left(y_{i}, y_{i-1}\right)=d_{i}}}^{\prime} \mathbb{P}_{x}\left[\bar{H}_{n}\left(y_{1}, \ldots, y_{i}\right)<a m(n)\right] \\
& \leq i ! i \sum_{d_{1}=1}^{g(n)} \sum_{\substack{y \in B_{n} \\
d(y, x)=d_{1}}} C a\left(2^{-n} m(n)+\xi_{n}\left(d_{1}\right)\right)\left(\sum_{d=1}^{n} \sum_{\substack{y \in B_{n} \\
d(y, x)=d}} C a\left(2^{-n} m(n)+\xi_{n}(d)\right)\right)^{i-1},
\end{aligned}
$$

which converges to 0 by (3.45) and (3.46). This finishes the proof of Proposition 3.1.

\section{Proof of Theorem 1.1 And of Theorem 1.2}

In this section we apply Theorem 1.3 to derive the asymptotic hitting distribution of randomly chosen sets in two different settings: for random clouds (namely we prove Theorem 1.1) and in the setting of drawing without replacement (which is Theorem 1.2).

Proof of Theorem 1.1. To prove Theorem 1.1 we will naturally show that the assumptions of Theorem 1.3 are satisfied for percolation clouds $A_{n}$ of density $\bar{m}(n)^{-1}$, where $n \log n \ll \bar{m}(n) \ll 2^{n}(\log n)^{-1}$.

We first verify condition (1.8), i.e. that $P$-a.s. $\left|A_{n}\right|=2^{n} \bar{m}(n)^{-1}(1+o(1))$. By Chebyshev exponential inequality, for any $\delta>0$ with $\lambda>0$,

$$
\begin{aligned}
P\left[\left|A_{n}\right| \gtrless(1 \pm \delta) 2^{n} n^{-1}\right] & \leq \exp \left\{\mp \lambda(1 \pm \delta) 2^{n} \bar{m}(n)^{-1}\right\}\left(1+\left(e^{ \pm \lambda}-1\right) \bar{m}(n)^{-1}\right)^{2^{n}} \\
& \leq \exp \left\{\mp \lambda(1 \pm \delta) 2^{n} \bar{m}(n)^{-1}+\left(e^{ \pm \lambda}-1\right) 2^{n} \bar{m}(n)^{-1}\right\} .
\end{aligned}
$$

Taking $\lambda$ sufficiently small and using the fact that $2^{n} \bar{m}(n)^{-1} \gg \log n$, we see that the right-hand side of the last equation is summable. Borel-Cantelli lemma then yields the result.

Let $f_{n}(k)=n(\log \bar{m}(n))^{-1} \mathbb{1}\{k=1\}+n \mathbb{1}\{k>1\}$. To prove that the first part of (1.11) is satisfied for the percolation cloud we show:

Lemma 4.1. There exists $C$ large enough, such that for a.e. realisation of $A_{n}$ and for $n$ large enough

$$
v_{n}(k) \leq C\left[\left(\begin{array}{l}
n \\
k
\end{array}\right) \bar{m}(n)^{-1}+f_{n}(k)\right] \quad \forall k \in\{1, \ldots, g(n)\} .
$$

Proof. Let $F_{n}(k)$ denote the right-hand side of (4.2). By definition of $v_{n}(k)$,

$$
\begin{aligned}
P\left[v_{n}(k) \geq F_{n}(k)\right] & \leq \sum_{x \in \mathcal{V}_{n}} P\left[\left|\left\{y \in A_{n}: d(x, y)=k\right\}\right| \geq F_{n}(k)\right] \\
& =2^{n} P\left[\left|\left\{y \in A_{n}: d(\mathbf{0}, y)=k\right\}\right| \geq F_{n}(k)\right] .
\end{aligned}
$$

Using the same calculation as in (4.1) this is bounded from above by

$$
2^{n} \exp \left\{-C \lambda\left[\left(\begin{array}{l}
n \\
k
\end{array}\right) \bar{m}(n)^{-1}+f_{n}(k)\right]+\left(e^{\lambda}-1\right)\left(\begin{array}{l}
n \\
k
\end{array}\right) \bar{m}(n)^{-1}\right\} .
$$

If we choose $\lambda=\log \bar{m}(n)$ and $C$ large enough for $k=1$, or $\lambda=$ const and $C$ large enough for $k>1$, then the right-hand side of the last equation decays at least as fast 
as $e^{-c n}$ for all $k$. Summing over $k$ and using Borel-Cantelli Lemma yields the desired result.

Lemma 4.1 implies that

$$
\sum_{k=1}^{g(n)-1} v_{n}(k) \xi_{n}(k) \leq \sum_{k=1}^{g(n)-1} C\left[\left(\begin{array}{l}
n \\
k
\end{array}\right) \bar{m}(n)^{-1}+f_{n}(k)\right] \xi_{n}(k) .
$$

Using Lemma 3.2(i),(iv) this can be bounded by

$$
C\left\{\sum_{k=1}^{g(n)-1} \xi_{n}(k)\left(\begin{array}{l}
n \\
k
\end{array}\right) \bar{m}(n)^{-1}+\sum_{k=1}^{3} f_{n}(k)\left(\begin{array}{l}
n \\
k
\end{array}\right)^{-1}+\sum_{k=4}^{g(n)-1} n f_{n}(k)\left(\begin{array}{l}
n \\
k
\end{array}\right)^{-1}\right\}
$$

The last two terms in the last formula are bounded by

$$
C \sum_{k=1}^{3} f_{n}(k) n^{-k}+C n^{3} n^{-4} \stackrel{n \rightarrow \infty}{\longrightarrow} 0
$$

as can be seen easily from the definition of $f_{n}(k)$. The first term in (4.6) equals

$$
\frac{n 2^{-n}}{2 \bar{m}(n)} \sum_{k=1}^{g(n)-1} \sum_{j=1}^{n-k}\left(\begin{array}{c}
n \\
j+k
\end{array}\right) \frac{1}{j}=\frac{n}{2 \bar{m}(n)} \sum_{j=1}^{n-1} \frac{2^{-n}}{j} \sum_{k=j+1}^{n \wedge(g(n)+j-1)}\left(\begin{array}{l}
n \\
k
\end{array}\right) \leq \frac{C n \log n}{\bar{m}(n)} .
$$

This tends to 0 by the assumptions on $\bar{m}(n)$. Therefore $v_{n}(k)$ verifies the first part of (1.11) $P$-a.s. .

To verify the second part observe first that if $\bar{m}(n) \geq \delta 2^{n} n^{-1}$ for some $\delta>0$, then (1.10) holds for $g(n)=2$. Therefore, by Lemma 4.1, $V_{n}(g(n)-1) \leq v_{n}(1)+1 \leq C \ll$ $\left|A_{n}\right|$. We can hence further suppose that $\bar{m}(n) \ll 2^{n} n^{-1}$. By moderate deviations argument, and since $n / 2-g(n) \gg n^{1 / 2}$,

$$
|\{y: d(\mathbf{0}, y) \leq g(n)-1\}| \leq 2^{n} e^{-c g(n)^{2} / n} .
$$

Since $2^{n} \bar{m}(n)^{-1} \gg n$ there is a function $f(n)$ such that

$$
2^{n} \bar{m}(n)^{-1} f(n) \gg n \quad \text { and } \quad 1 \gg f(n) \gg e^{-c g(n)^{2} / n} \text {. }
$$

As in (4.1)

$$
P\left[V_{n}(g(n)-1) \geq \frac{2^{n} f(n)}{\bar{m}(n)}\right] \leq 2^{n} \exp \left\{\frac{2^{n}}{\bar{m}(n)}\left[-\lambda f(n)+\left(e^{\lambda}-1\right) e^{-c g(n)^{2} / n}\right]\right\} .
$$

For our choice of $f$ this is summable. Therefore a.s. for $n$ large enough $V_{n}(g(n)-1) \leq$ $2^{n} \bar{m}(n)^{-1} f(n) \ll\left|A_{n}\right|$. This verifies the second part of (1.11).

We have verified that with $P$ probability one the sequence of percolation clouds $A_{n}$ satisfies all the assumptions of Theorem 1.3, This proves Theorem 1.1.

Proof of Theorem 1.2. For any $m(n)$ satisfying the conditions of Theorem 1.2 it is possible to choose $\bar{m}(n)$ satisfying the conditions of Theorem 1.1 such that

$$
\bar{m}(n)^{-1} \geq(1+\varepsilon) m(n) .
$$

We now consider a sequence of percolation clouds $A_{n}$ with density $\bar{m}(n)^{-1}$ defined on the same probability space $\left(\Omega^{\prime}, F^{\prime}, P^{\prime}\right)$ as $A_{n}^{\prime}$. Since $P^{\prime}$-a.s. for all $n$ large enough $\left|A_{n}\right|>2^{n} m(n)^{-1}=\left|A_{n}^{\prime}\right|$, we can couple $A_{n}^{\prime}$ and $A_{n}$ in the way that for all $n$ large $A_{n}^{\prime} \subset A_{n}$. Moreover, $A_{n}$ satisfies the conditions (1.9)-(1.11) of Theorem 1.3. To finish 
the proof observe that if $A_{n}$ satisfies these conditions, then any subset of $A_{n}$ satisfies them too.

Acknowledgements. Both authors thank the Chair of Stochastic Modelling of the École Polytechnique Fédérale of Lausanne for financial support. Jiří Černý thank the Centre de Physique Théorique of Marseille for hospitality.

\section{REFERENCES}

[AS72] Milton Abramowitz and Irene A. Stegun, Handbook of mathematical functions with formulas, graphs, and mathematical tables., National Bureau of Standards Applied Mathematics Series, 55, fifth edition, John Wiley \& Sons Inc., Washington, D.C., 1972.

[BB02] E. Bertin and J.-P. Bouchaud, Dynamical ultrametricity in the critical trap model, J. Phys. A: Math. Gen. 35 (2002), 3039.

[BBG03a] Gérard Ben Arous, Anton Bovier, and Véronique Gayrard, Glauber dynamics of the random energy model. I. Metastable motion on the extreme states, Comm. Math. Phys. 235 (2003), no. 3, 379-425.

[BBG03b] Gérard Ben Arous, Anton Bovier, and Véronique Gayrard, Glauber dynamics of the random energy model. II. Aging below the critical temperature, Comm. Math. Phys. 236 (2003), no. 1, 1-54.

[BČ06a] Gérard Ben Arous and Jiří Černý, The arcsine law as a universal aging scheme for trap models, to appear in Comm. Pure Appl. Math. (2006).

[BČ06b] Gérard Ben Arous and Jiří Černý, Dynamics of trap models, École d'été de physique des Houches, Session LXXXIII, Mathematical Statistical Physics, Elsevier, 2006, pp. 331-394.

[BEGK01] Anton Bovier, Michael Eckhoff, Véronique Gayrard, and Marcus Klein, Metastability in stochastic dynamics of disordered mean field models, Prob. Theor. Rel. Fields (2001), no. 119, 99-161.

[BEGK02] Anton Bovier, Michael Eckhoff, Véronique Gayrard, and Markus Klein, Metastability and low lying spectra in reversible Markov chains, Comm. Math. Phys. 228 (2002), 219-255.

[BG06] Gérard Ben Arous and Véronique Gayrard, Elementary potential theory on the hypercube, preprint math.PR/0611178, 2006.

[Dia88] Persi Diaconis, Group representations in probability and statistics, Institute of Mathematical Statistics Lecture Notes-Monograph Series, 11, Institute of Mathematical Statistics, Hayward, CA, 1988.

[Fel71] William Feller, An introduction to probability theory and its applications. Vol. II., Second edition, John Wiley \& Sons Inc., New York, 1971.

[Kem61] J.H.B. Kemperman, The passage problem for a stationary markov chain, Satistical Reseach Monographs, Vol. I, University of Chicago Press, Chicago, 1961.

[Mat88] Peter Matthews, Covering problems for Markov chains, Ann. Probab. 16 (1988), no. 3, $1215-1228$.

[Mat89] Peter Matthews, Some sample path properties of a random walk on the cube, J. Theoret. Probab. 2 (1989), no. 1, 129-146.

JiŘí Černý, École Polytechnique Fédérale de Lausanne, 1015 Lausanne, Switzerland E-mail address: jiri.cerny@epfl.ch

Véronique Gayrard, Laboratoire D'Analyse, Topologie, Probabilités, CMi, 39 rue Joliot-Curie, 13453 Marseille Cedex

E-mail address: gayrard@latp.univ-mrs.fr, veronique@gayrard.net 